\title{
Designing a simulation-supported learning process for decision makers in the Mekong region
}

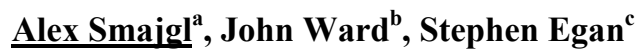 \\ ${ }^{a}$ CSIRO Ecosystem Sciences, Townsville 4810 Queensland, Australia \\ Email: alex.smajgl@,csiro.au \\ ${ }^{b}$ CSIRO Ecosystem Sciences, Dutton Park 4102 Queensland, Australia \\ ${ }^{c}$ CSIRO Ecosystem Sciences, Highett 3190 Victoria
}

\begin{abstract}
Hydropower development, expansion of rubber plantations, large scale irrigated agriculture and sea level rise represent some of the key opportunities and risks perceived by decision makers in the Mekong region. Using opportunities wisely and responding effectively to risks without compromising a sustainable future demands insights into complex system responses. This paper describes a participatory learning process for decision makers and decision influencers in five countries located in the wider Mekong region. Structured engagement processes and mixed methods address the learning challenges associated with high levels of system complexity and the values of competing interests. The mixed methods approach involved a suite of disciplinary specific models, household surveys and an agent-based simulation model. Learning was facilitated by challenging beliefs articulated by stakeholders during the participatory process. In the context of the wider Mekong region modelling was an essential element and this paper is focused on the role of agent-based modelling and its effectiveness. A key insight from this study is that the potential to introduce doubt and challenge prevailing beliefs - and thereby to facilitate learning - increases with the complexity of the particular method implemented. Overall, this work emphasises the relevance of process design to effectively address cognitive barriers encountered at the science-policy interface and achieve targeted learning outcomes.
\end{abstract}

Keywords: Agent-based modelling; Participatory process design; Mekong 


\section{INTRODUCTION}

Delivering research into applied decision making situations can be challenging if the issues are highly complex. The task becomes even more challenging when the decision context is characterized by highly contested values. David Hume famously argued that normative or "ought" statements are not necessarily derived from research facts and reason or "is" statements (Cohen, 2010; Hume, 1739). Complexity introduces a gap between decision-making and science thus undermining the ability for research insights to be understood and implemented. Contested values create biases in stakeholders' perception so that research results might further polarise the policy debate instead of facilitating an evidence-based dialogue. Overcoming these two key challenges requires (1) an effective process to bridge between science and policy and (2) a sophisticated set of methods that helps decision makers consider higher levels of complexity without subverting the science-policy interface.

Participatory research principles are often identified to facilitate system learning (Smajgl and Ward, in print) or to resolve conflicts (Barreteau et al., 2010). Voinov and Bousquet (2010), Lynam et al. (2007), and Barreteau et al. (2010) provide essential overviews for the domain of participatory modelling. The process protocol implemented for this paper does not necessarily require modelling. However, in the context of the wider Mekong region modelling was an essential element and this paper is focused on the role of agent-based modelling and its effectiveness. Unfortunately, the number of publications outlining and testing particular design protocols for participatory research or participatory modelling is limited (Gaddis et al., 2009; Smajgl, 2010; Jones et al., 2009). The most rigorous effort was published by research groups involved with Companion Modelling, a process that largely integrates role-playing games and agent-based modelling and aims for conflict resolution (Barreteau, 2003; Bousquet et al., 2007; Perez et al., 2011). Ideally, a variety of designs for participatory research with precise engagement protocols would be available to researchers, and selections based on evaluations of each design across a diverse range of contexts. Such a situation would allow an effective cross-comparative analysis to identify which steps and methods are most suitable in which context. Unfortunately, science is still far away from this ideal and it will take the coordinated effort of many teams to develop an improved understanding of process design options (Poteete et al., 2010; Smajgl and Ward, in print).

The role of process design gains importance with increasing complexity as in highly complex situations the risk is very high that the science-policy interface remains dysfunctional. Methods need to be identified for analysing complex interactions while providing sufficient transparency and ability to abstract simple insights. In cases where complexity emerges from the interaction of humans within their environment, agent-based modelling is often chosen as it provides scientists with the methodology to simulate household behaviour explicitly and consider social and ecological interactions and their consequences in a spatial environment (Matthews et al., 2007; Troitzsch, 2013).

The process protocol presented and evaluated in this paper does not involve stakeholders in the design of the model. On the contrary, as the aim is to facilitate stakeholder learning this approach avoids mapping stakeholder assumptions into the model. Instead, primary data and experts are consulted to structure, parameterize and calibrate the agent-based model. As a result, model results can be very different from causal beliefs held by relevant stakeholders, which provides the potential to challenge existing beliefs and facilitate system learning. Participatory elements include that stakeholders design the overall project, specify what results and metrics are required (incl. determining the model use), and guide research direction from workshop to workshop.

This paper describes the third generation of a learning-focused participatory process design. Previous versions (Smajgl, 2010; Smajgl et al., 2009) have been refined and the revised protocol was implemented in the wider Mekong region. This implementation involves cross-sectoral and transboundary coordination in a highly contested and highly complex context, feeding into five particular decision making situations in five countries. The cross-scale issue is of particular relevance as the selected decision making options are likely to have regional (transboundary) consequences while being located at the sub-national level. These decisions include hydropower, irrigation schemes, plantations, and adaptation to sea level rise. Stakeholders identified poverty as the key indicator for assessing relevant decision making options. Facilitating a learning process for decision makers at district, province, national and supra-national levels requires overcoming wrong perceptions and improved coordination between relevant levels of governance. This work presents how agent-based modelling and other methods were used in a learning-focused participatory process. The following Section provides the necessary context for the wider Mekong region, followed by the design protocol and results. 


\section{MEKONG REGION CONTEXT}

The Greater Mekong Subregion comprises of Vietnam, Cambodia, Lao PDR, Thailand, Myanmar and China's Province Yunnan. The region is currently experiencing an unprecedented influx of private and public investment, largely establishing hydropower, rubber plantations, irrigation schemes for food and energy crops, and mining (Smajgl and Ward, 2013). The majority of current investments demote development and poverty alleviation to secondary side-effects as primary interests are focused on expanding profit margins, securing access to natural resources, and safe-guarding national energy and food security. Understanding unintended side-effects of targeted investments in the Mekong region is critical if decision makers aim to achieve poverty alleviation targets. An effective understanding can allow for selecting and amending investments to safeguard or improve the livelihoods of the poor. Due to the complexity of this task and the relevance of human behaviour in the form of household responses to potential changes in their socioeconomic and bio-physical environment, agent-based modelling was chosen to contribute to a learning process with decision makers (Foran et al., in print; Smajgl et al., in review; Smajgl and Ward, in review; Smajgl and Ward, in print).

\section{PARTICIPATORY RESEARCH DESIGN FOR THE MEKONG REGION}

\subsection{The Challenge and Reconstruct Learning method}

Improving decision makers' understanding of what possible implications large-scale changes might have in the Mekong region requires an approach that effectively bridges research and policy. This work aims to achieve such system learning by implementing the ChaRL framework (see the schematic in Figure 2) (Smajgl and Ward, in print). The framework entails five key steps to facilitate learning, accomplished via a structured and sequenced set of facilitated participatory workshops.

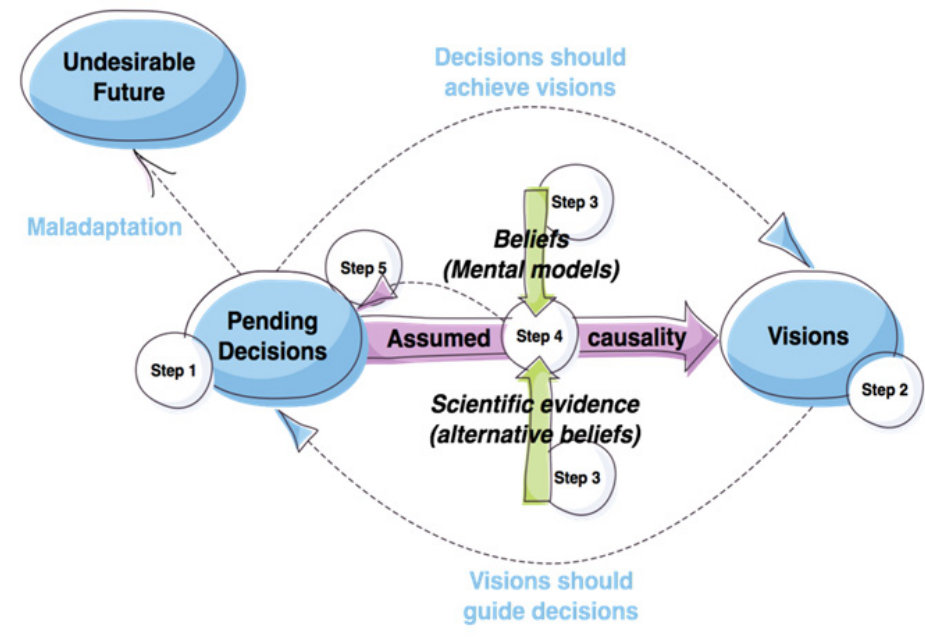

Figure 1 The ChaRL framework - Challenge and Reconstruct Learning (Smajgl and Ward, in print)

The five ChaRL steps define the structured process of formally questioning and measuring existing underlying assumptions (heuristics) and reconstructing revised assumptions within the understanding of a larger systems view. We understand such rational reconstruction in the tradition of Habermas (2005) as the key process of learning, which is facilitated as an exchange of intuitive knowledge.

Step 1 scopes out the context with the objectives, including the decision making context and options, and the relevant success indicators as perceived by the decision makers. Inviting the relevant decision makers to codesign the research is critical to ensure high levels of engagement (Smajgl, 2010).

In step 2 visions for a specified geographic location are developed as narratives of plausible futures desirable for all relevant stakeholders (Foran et al., in print). This step might need to be implemented in multiple iterations if the set of decisions are likely to affect multiple action arenas (i.e. scales), which demand separate facilitation. The iterative approach involves the presentation of visions from other locations or scales and invites the revision of the original vision draft, accounting for external implications and consequences.

During step 3 scientific evidence is presented to assess expected outcomes of contemplated decisions. During this step note takers formally record existing beliefs, expressed as statements that articulate perceived causal 
relationships held by decision makers. Note takers are trained in identifying statements that constitute causal beliefs and record them in a systematic and targeted way.

Step 4 constitutes the core learning step as previously recorded beliefs are explicitly compared (a) with each other and (b) with scientific evidence. Revealed contrasts are discussed in the context of how pending decisions will contribute (or not) to the realisation of desirable visions articulated in Step 1. This process facilitates the reconstruction of underpinning heuristics and assumptions (the set of beliefs) against the backdrop of desired futures. The process aims to guide the discussion session towards an action plan that first accounts for revealed disparities between beliefs and desired futures, and second has sufficient promise to achieve the desired future and avoid non-desired, mal-adaptive outcomes.

Step 5 constitutes a specific set of actions to realise the adaptation and development pathway participants desire. A comprehensive monitoring and evaluation protocol extends and completes the ChaRL framework (Smajgl and Ward, in review).

\subsection{Multi-level engagement in the wider Mekong region}

The ChaRL framework was implemented in a participatory project in the wider Mekong region, involving decision makers from district, province, and central Government as well as from supra-national agencies. After an eight-month scoping mission that allowed decision makers from five countries to select one decision making context, visions were drafted in each of the five local study areas. In addition to these five parallel 'local' processes (that involved stakeholders from the district to the central Government an additional process brought together decision makers operating at the supra-national level who were largely interested in the wider Mekong region. These visions were based on drivers that participants perceived to be of high impact and highly uncertain (Foran et al., in print). However, due to the connectivity within the wider Mekong region, changes in some areas are likely to impact on other areas, shifting boundaries perceived in the envisioning process. Based on draft visions from all six processes participants revised their vision in a second round of workshops.

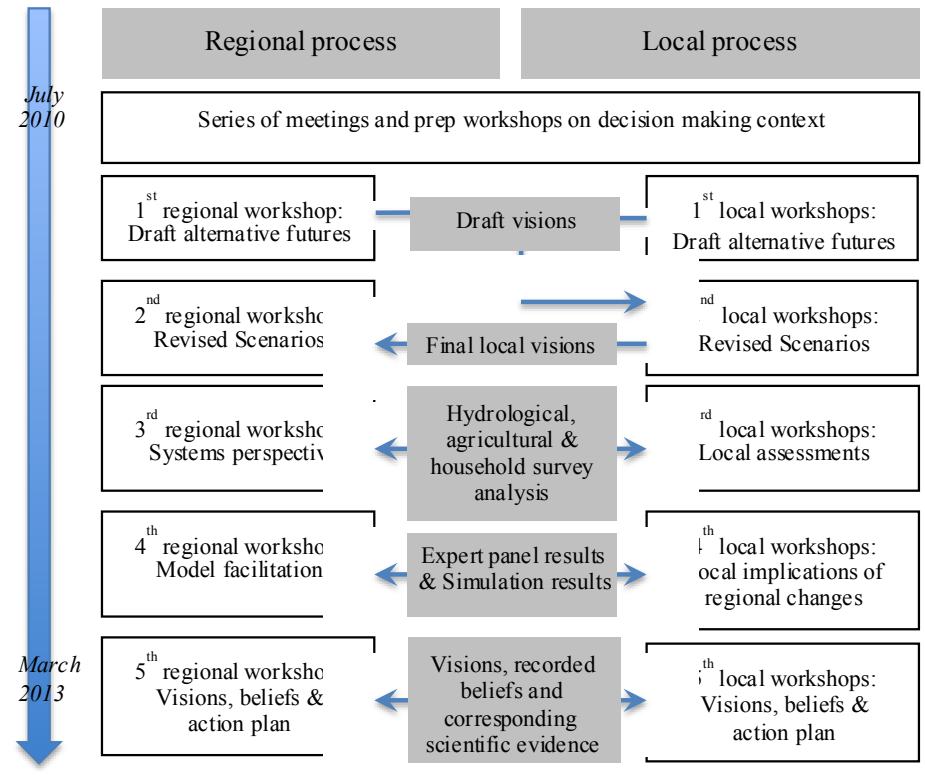

Figure 2 The ChaRL framework - Challenge and Reconstruct Learning (Smajgl and Ward, in print)

The third round of workshops presented scientific evidence emerging from hydrological and agricultural work implemented in the first twelve months. On the second day household survey results were presented. All results were presented as draft results and therefore as 'alternative beliefs'. Break-out group discussions were captured by trained note takers to elicit belief-type statements. Each break-out group had a note taker allocated and all statements that articulated causal relationships were recorded on specific sheets to ensure a structured elicitation.

The fourth workshop presented integrated assessments in the form of results generated by a multidisciplinary expert panel (Smajgl and Ward, 2013) and the agent-based modelling results. Also during this round of workshops beliefs were recorded by observers in break-out group discussions. The final workshop 
presented recorded beliefs and matching scientific evidence to create challenges against the backdrop of the visions participants created during the first year.

\subsection{The role of agent-based modelling}

The psychological foundation of this participatory process assumes that prevailing heuristics can be revealed, and that they do not adequately represent the reality of the decision making context. Challenging these heuristics with a singular reliance on scientific evidence can be ineffective if complexity is very high and participants perceive a large gap between what is being presented and the ensemble of their existing beliefs. Thus we first introduce the results of disciplinary models as they normally include lower levels of complexity. Concurrently the majority of the results presented from hydrological or agricultural models corresponded with existing beliefs. However, the presentation of household survey results pointed to an increased potential to challenge existing beliefs, as some of the presented evidence contradicted recorded beliefs. The final sessions presented the results from agent-based simulations, introducing the dynamic representation of likely household responses in addition to the feedbacks within the social-ecological system. This methodology provided the largest potential for challenging preeminent beliefs.

This is not surprising as the approach aims to test the generalisations and simplifications participants have constructed based on their own observations and from their interactions with experts. Many of these simplifications correspond with scientific assessments and analysis for some areas, but if generalised location-specific observations appear to create disconnects between proposed actions and stated objectives. Naturally, methods that increase the number of interacting processes and therefore the level of complexity have a larger potential to challenge simplifying heuristics and assumptions. Two additional factors enhance the ability of agent-based modelling to challenge beliefs. First we found that an important domain for misconceptions among decision makers is their expectation of household behaviours. Second is the spatial distribution of results, revealing that certain measures might be successful in some areas but not in others.

Implementing the ChaRL protocol in the Mekong harnessed the strength of agent-based modelling by presenting results from Monte-Carlo runs. Live model runs were limited to a few sessions to accentuate the spatial aspects of critical indicators. For instance dynamic poverty distribution maps helped to emphasise that poverty is a dynamic seasonal phenomenon and that poverty is critically linked to migration and that homogenous changes in poverty levels in all areas is unlikely.

It is critical to emphasise that this process leaves it to stakeholders to identify their own misconceptions. The agent-based model is only utilised to seed doubt and stakeholders are asked to validate the results and, thereby to question their own beliefs. Model results are at no stage presented as predictions. The debate among stakeholders helped identifying the nature of existing misconceptions and revealed generalisations.

\section{EXPERIENCES, RESULTS AND LESSONS LEARNT}

This Section provides three kinds of insights. First, the experiences with the ChaRL process are summarized. Second, the learning results are presented and third, the lessons learnt are summarized.

\subsection{Experiences with scientific team and participants}

Two types of experiences can be distinguished, experiences within the team of collaborating scientist and those with actual participants. One of the key challenges for a participatory process as described above is locating and validating the research design within the dominant modus operandi of traditional science approaches. The majority of scientists involved in this study were struggling with the additional burden required to rigorously implement the design of the participatory process. Traditional processes do not generally accommodate the initial step that allows participating stakeholders to design the project, determine primary research foci and the attendant metrics. Allowing research ownership to develop requires time, introduces uncertainty for researchers, and is often perceived as an unnecessary resource burden. Visions, on the other hand, were perceived as a very valuable contribution amongst participating scientists. Instructions to scientists to emphasise inherent uncertainties in research results (uncertainty often invites stakeholders to articulate their own beliefs), in contrast to attempting to convince stakeholders, was largely interpreted as undermining credibility. By the end of the process, most contributing scientists saw the pieces coming together and provided positive feedback. However, scientists criticised the focus of contrasting existing beliefs and scientific evidence without revisiting detailed methodological discussions.

Participants experienced the process largely very positively, as they were instrumental in project inception, designing the project proposal and hence the research foci. The creation of visions was challenging for many 
participants as conflicting values had to be brought together from multiple, often competing sectors. However, the introduction of narrative visions from other Mekong locations was for many the most interesting step of the process. The third and fourth science-focused workshops created a mixed experience for decision makers. First, the time allocated to discussions concerned with technical details was considered as excessive. In contrast, the benefits of the time dedicated to break-out group discussions were emphasised, as this allowed an improved appreciation and understanding of other stakeholder perceptions and the science insights. The final workshop provided surprises in most cases and feedback from participants was overwhelmingly positive. However, in countries without democratic structures we experienced additional challenges. For instance, in Vietnam the direct challenging of existing beliefs was too provocative as one of the findings highlighted the policy conflict between two central ministries. Four high-level participants dominated the fifth workshop, prohibiting step 5 (see 0 ) to eventuate. Thus, a sixth workshop was conducted at a more technical level, discussing land use change and strategies to achieve more sustainable outcomes. The shift towards more technical aspects (symptom) and away from the political perspective (cause) provided the key to overcome critical barriers.

\subsection{Learning-related results}

Approximately 150 beliefs were recorded during the workshops, many of which did not correspond with the research foci initially defined by stakeholders. This group of beliefs included beliefs regarding, for instance globalization, tourism, and ethnicities. Of the remaining 70 documented beliefs, the majority were not contradicted by the scientific evidence produced as part of the Exploring Mekong Region Futures project. Twenty-four beliefs were identified that were not aligned with the project's scientific evidence and could therefore be challenged. Based on the responses by stakeholders the most surprising contradictions were linked to the following beliefs:

- Investments in large-scale irrigation schemes helps in alleviating poverty (found in Northeast Thailand, and in the Nam Ngum catchment, Lao PDR)

- Payments for ecosystem services are generally an effective way to achieve conservation goals (found in Xishuangbanna, Yunnan, China)

- The collapse of fish stocks in the Tonle Sap will cause substantial out-migration (found in the Tonle Sap area, Cambodia)

- Soft measures (i.e. modifications to cropping patterns) have a low potential to alleviate the consequences of sea-level rise (found in the Vietnam's Mekong Delta)

These beliefs have a critical influence on development decisions in the Mekong region. Providing counterintuitive evidence created some major shifts in thinking that is likely to amend future investments.

\subsection{Lessons learnt}

A few lessons can be articulated as a corollary of the experiences expressed by participants and the evaluation of project results. First, the general trajectory of the ChaRL framework seems very promising in achieving learning among decision makers and decision influencers, particularly when contending value propositions are central to final resolutions. Second, in complex and interdependent decision arenas, agentbased modelling in conjunction with household surveys provides an effective method for challenging existing beliefs. Third, researchers involved in the project require intensive preparation, for instance in form of roleplaying games, to understand the methodology. Fourth, when democratic structures are absent from political systems, the engagement process might be more effective if the presentation of belief-science contrasts is softened and approached from a solution perspective instead of the problem domain. Fifth, effective strategies need to be found to ensure high retention rates among workshop participants as otherwise an insufficient number of delegates in the final workshop 5 identify with the visions created in workshops 1-2.

\section{DISCUSSION}

Adding the design of engagement processes to the domains of scientific thinking seems imperative for situations with high levels of complexity and highly contested values. Testing the effectiveness of variants of process designs is resource demanding and requires the contributions of multiple teams to reveal which process steps and methods are best suited to creating an effective policy-science interface (Poteete et al., 2010). This study aims to make a contribution to this emerging research field.

Agent-based modelling has been tested in three variants of the ChaRL framework and in all cases it has provided the largest impact by revealing essential insights into prevailing system complexities. At the same time, agent-based modelling allowed for an effective means to translate insights into simple and coherent 
policy messages. However, the increasing number of system dynamics incorporated by the model requires efforts to maintain simple, easily communicated model functions and avoid the perceptions of a black box. Retaining confidence among stakeholders is a key challenge with all complex systems modelling. In synthesis, the increased complexity of the decision arena introduces the opportunity to facilitate new insights and learning, but this is counterbalanced by the challenge to maintain high levels of confidence among stakeholders (particularly in situations with highly contested values).

The tension between opportunity and scepticism emphasizes the need for a thoroughly designed engagement process to initiate a scaffolding of trust and ownership, critical facets that enable more complex methods such as agent-based modelling to make a realisable contribution to the science-policy interface.

\section{REFERENCES}

Barreteau, O., 2003. The joint use of role-playing games and models regarding negotiation processes: characterization of associations. JASSS - The Journal of Artificial Societies and Social Simulation 6(2) $<$ http://jasss.soc.surrey.ac.uk/6/2/3.html\%3E.

Barreteau, O., Bots, P.W.G., Daniell, K.A., 2010. A Framework for Clarifying "Participation" in Participatory Research to Prevent its Rejection for the Wrong Reasons. Ecology and Society 15(2) 1.

Bousquet, F., Castella, J.C., Trebuil, G., Barnaud, C., Boissau, S., Kam, S.P., 2007. The use of multi-agent systems in a companion modeling approach for agroecosystem management in Southeast Asia. Outlook in Agriculture 36(1) 57-62.

Cohen, R., 2010. Hume's moral philosophy, Stanford Encyclopaedia of Philosophy.

Foran, T., Ward, J., Kemp-Benedict, E., Smajgl, A., in print. Developing detailed foresight narratives: a participatory technique from the Mekong region. Ecology and Society.

Gaddis, E.J.B., Harp Falk, H., Ginger, C., Voinov, A., 2009. Effectiveness of a participatory modeling effort to identify and advance community water resource goals in St. Albans, Vermont. Environmental Modelling and Software doi:10.1016/j.envsoft.2009.06.004.

Habermas, J., 2005. Concluding comments on empirical approaches to deliberative politics. Acta Politica 40 (3) $384-392$.

Hume, D., 1739. A treatise of human nature. John Noon, London.

Jones, N.A., Perez, P., Measham, T.G., Kelly, G.J., d'Aquino, P., Daniell, K.A., Dray, A., Ferrand, N., 2009. Evaluating Participatory Modeling: Developing a Framework for Cross-Case Analysis. Environmental Management, 44(6): 1180-1195.

Lynam, T., de Jong, W., Shell, D., Kusumanto, T., Evans, K., 2007. A review of tools for incorporating community knowledge, preferences, and values into decision making in natural resources management. Ecology and Society 12(1) 5.

Matthews, R., Gilbert, N., Roach, A., Polhill, J., Gotts, N., 2007. Agent-based land-use models: a review of applications. Landscape Ecology 22(10) 1447-1459.

Perez, P., Aubert, S., Dare, W., Ducrot, R., Jones, N., Queste, J., Trebuil, G., Van Paassen, A., 2011. Assessment and monitoring of the effects of the ComMod approach, In: Etienne, M. (Ed.), Companion modelling: a participatory approach to support sustainable development. Editions Quae: Versaille Cedex.

Poteete, A.R., Janssen, M.A., Ostrom, E., 2010. Working togeher: collective action, the commons, and multiple methods in practice. Princeton University Press, Princeton.

Smajgl, A., 2010. Challenging beliefs through multi-level participatory modelling in Indonesia. Environmental Modelling and Software 25(11) 1470-1476.

Smajgl, A., Foran, T., Dore, J., Ward, J., Larson, S., in review. Visions, beliefs and transformation: Methods for understanding cross-scale and trans-boundary dynamics in the wider Mekong region. Global Environmental Change.

Smajgl, A., Morris, S., Heckbert, S., 2009. Water policy impact assessment - combining modelling techniques in the Great Barrier Reef region. Water policy 11 191-202.

Smajgl, A., Ward, J., 2013. The Water-Food-Energy Nexus in the Mekong Region. Springer: New York.

Smajgl, A., Ward, J., in review. A design protocol for research impact evaluation: Development investments of the Mekong region. Research Evaluation.

Smajgl, A., Ward, J., in print. A framework for bridging Science and Decision making. Futures.

Troitzsch, K.G., 2013. Historical Introduction, In: Edmonds, B., Meyer, R. (Eds.), Simulating Social Complexity. Springer: Berlin, Heidelberg, pp. 13-21.

Voinov, A., Bousquet, F., 2010. Modelling with stakeholders. Environmental Modelling and Software 25(11) 1168-1281. 